\title{
Expulsion : genèse et pratique d'un contrôle en Allemagne (partie 3)
}

\section{Frank Paul Weber}

\section{OpenEdition \\ Journals}

\section{Édition électronique}

URL : http://journals.openedition.org/conflits/627

DOI : $10.4000 /$ conflits.627

ISSN : $1777-5345$

Éditeur :

CCLS - Centre d'études sur les conflits lilberté et sécurité, L'Harmattan

\section{Édition imprimée}

Date de publication : 15 octobre 1996

ISSN : 1157-996X

\section{Référence électronique}

Frank Paul Weber, «Expulsion : genèse et pratique d'un contrôle en Allemagne (partie 3) », Cultures \& Conflits [En ligne], 23 I automne 1996, mis en ligne le 15 mars 2006, consulté le 30 mars 2021. URL : http://journals.openedition.org/conflits/627 ; DOI : https://doi.org/10.4000/conflits.627

Ce document a été généré automatiquement le 30 mars 2021.

Creative Commons License 


\title{
Expulsion : genèse et pratique d'un contrôle en Allemagne (partie 3)
}

\author{
Frank Paul Weber
}

La contrainte extérieure Les accords d'admission, de réadmission de personnes expulsées entre Etats ne sont pas en soi un effet tardif de politiques migratoires confrontées à des flux massifs de migrants. Dans le cas de l'Allemagne, ces accords sont consubstantiels à l'idée de procéder à des expulsions. L'Empire russe et la Prusse conclurent ainsi tout au long du XIXe siècle des conventions bilatérales permettant l'extradition de leurs déserteurs et criminels respectifs ${ }^{1}$. Les « crimes » sanctionnés par cette mesure incluaient cependant les simples faits d'absence de ressources propres, de vagabondage ou de défaut de papiers d'identité ${ }^{2}$.

On peut distinguer trois ou quatre types d'accords. En premier lieu les accords de réadmission signés dans les années 1950 et 1960: l'Allemagne et ses partenaires s'engagent à reprendre leurs ressortissants (expulsés) dotés de papiers d'identité (permis de conduire, carte d'identité, passeport...). L'identité est plus ou moins attestée par un document officiel. Déjà apparaissent des clauses permettant l'expulsion de ressortissants de pays tiers entrés illégalement dans un des pays signataires de l'accord. A la suite de l'« ouverture » des frontières des pays du Pacte de Varsovie, l'Allemagne s'est efforcée de conclure avec ces "nouveaux» voisins à l'Est des accords de réadmission concernant surtout les ressortissants de pays tiers. Il s'agit d'accords avec des pays de transit ayant cessé d'être, souvent depuis peu, comme la Pologne, des pays d'émigration importante vers l'Allemagne ou ne l'ayant jamais été à l'instar de la Suisse. Alors que les accords de réadmission avec les pays de transit ont, pour ainsi dire, épuisé le «champ du possible » en la matière, les accords anciens ou récents couvrant toutes les possibilités de transit terrestre, le troisième type d'accords de réadmission, ceux avec les pays d'origine, est par essence une catégorie vouée à s'élargir à l'infini ou presque, puisqu'il peut être conclut autant d'accords qu'il y a d'Etats sur la carte du monde susceptibles de "produire " un flux de migrants vers l'Allemagne. La plupart de ces accords concernent des pays du Sud-Est de l'Europe : Roumanie, Bulgarie, Croatie (voire l'accord avec la Turquie sur le respect des droits de l'homme en cas d'expulsion). Un accord avec le Vietnam, dont la mise en œuvre (ou 
plutôt sa quasi non-application) mécontente Bonn, a été signé en 1995. Un accord de réadmission avec l'Algérie est négocié depuis plusieurs années. Enfin on pourrait ajouter une dernière catégorie à celles concernant les accords bilatéraux : les accords multilatéraux, notamment ceux de la complexe configuration Schengen... ${ }^{3}$. Ces divers accords de réadmission fonctionnent en définitive comme un sous-ensemble institutionnel $\mathrm{du}$ dispositif d'expulsion. Il apparaît en effet que ces accords se complètent, se neutralisent, s'influencent mutuellement. Cela est vrai non seulement en termes de "flux d'expulsion" (les tenaces opposants - les mêmes en étant aussi indirectement les promoteurs - de l'idée d'« Europe forteresse » évoquent la possibilité d'expulsions en chaîne, sorte de théorie des dominos de l'expulsion) mais aussi en termes de codes, de catégories juridiques. Hormis la possibilité d'expulsions successives à partir d'accords bilatéraux de réadmission s'emboîtant comme des poupées russes ${ }^{4}$, il faut également mentionner que les accords avec les pays de transit ont tendance à perdre de leur importance en raison du recours prioritaire aux accords avec les pays d'origine par les autorités allemandes. Ainsi il n'est pas si étonnant que sur la base de l'accord de 1993 signé avec la Pologne moins de 6000 personnes ont été, chaque année, expulsées vers la Pologne en 1993 et 1994, alors que ce même accord avait prévu un «seuil plafond» de 10000 personnes renvoyées vers la Pologne pour la seule année 1993. Si un renvoi massif de ressortissants de pays tiers vers la Pologne n'a pas eu lieu, c'est en effet à cause de l'existence et de l'utilisation d'accords de réadmission avec les pays d'origine fournissant les contingents les plus nombreux de migrants entrant illégalement en Allemagne par la frontière germano-polonaise. L'accord avec la Roumanie $^{5}$ est en effet antérieur à celui avec la Pologne, l'accord avec la Bulgarie étant intervenu en $1994^{6}$. Les Roumains ayant transité par la Pologne puis expulsés directement vers la Roumanie (souvent par charter à partir de l'aéroport berlinois de Schönefeld) sont autant de personnes que les autorités allemandes ne cherchent pas à transférer à leurs alter ego polonais. Il faut d'ailleurs remarquer qu'il s'agit là d'une stratégie délibérée et non le fruit de situations pour lesquelles un renvoi direct en Roumanie serait plus aisé : la plupart des ressortissants roumains ou présumés tels sont en fait appréhendés dans la zone frontalière germano-polonaise mais transférés ensuite à l'intérieur du pays (soit Berlin) afin d'y être expulsés ${ }^{7}$. La formulation de ces différents accords témoigne à la fois de l'évolution du discours juridique en la matière et de la grande flexibilité qu'il peut conférer. On peut ainsi constater qu'aujourd'hui l'accent est mis sur l'expulsion de personnes séjournant illégalement en Allemagne alors que les accords des années cinquante et soixante insistaient plutôt sur l'entrée illégale, le franchissement non-autorisé de la frontière (acte à partir duquel les délais étaient fixés). Les accords de 1955 et 1993 signés avec la Suisse en sont le meilleur exemple puisque l'intitulé de l'accord passe de «Admission de personnes à la frontière » (1955) à « Réadmission de personnes "au séjour non-autorisé" » (1993). De la même manière l'accord avec le Vietnam prévoit en premier lieu la réadmission, jusqu'à l'an 2000, par le Vietnam de ressortissants vietnamiens résidant déjà illégalement en Allemagne à raison de quotas annuels, ainsi que les Vietnamiens qui, à l'avenir, pourraient entrer sur son territoire et faire l'objet d'un arrêté d'expulsion. Par ailleurs l'énoncé des critères retenus pour attester de la nationalité d'une personne expulsable est, tout au long de l'historique de ces accords, l'objet d'un obscurcissement volontaire. Les accords des années 1990 systématisent en effet des énoncés antérieurs comme « La nationalité pourra également être présumée à l'aide d'autres moyens » (Accord francoallemand de 1960, Art. 1 \& 5) ou les « indices » [Anhaltspunkte] permettant de vérifier 
la nationalité de la personne à expulser (accords avec la Suisse et la Norvège en 1955). Les plus récents accords requièrent seulement que la nationalité (ou parfois le franchissement illégal de la frontière) soit "rendue crédible " [glaubhaft machen], précisant parfois par quels biais (langue, témoignages, certificats d'assurance, permis de conduire...). Dans la relation de pouvoir à l'extérieur Etat expulsant/Etat d'origine ou de réadmission (qui double celle, de l'intérieur, agences de contrôle/individus expulsables-expulsés), la "stratégie de pouvoir » ne va pas sans résistance : l'expulsion n'étant plus un seul acte unilatéral, l'Etat allemand se doit de négocier avec d'autres Etats le retour de leurs ressortissants. Les tentatives d'expulsions massives de l'immédiate après Première Guerre Mondiale furent ainsi largement limitées par le refus des pays d'origine, particulièrement la Pologne, de réadmettre sur leur territoire les expulsés de l'Empire allemand'. Aussi, la signature d'accords de réadmission avec plusieurs pays était, dès les années 1920, considérée comme une nécessité par les autorités prussiennes, comme en témoigne une lettre de décembre 1922 du Ministre prussien de l'Intérieur, Severing, au Ministre de l'Intérieur du Reich, dans laquelle est évoquée la conclusion de semblables accords, en particulier avec la Pologne, la Russie, la Lettonie, la Lithuanie et l'Estonie ${ }^{9}$. Sous la République de Weimar, aucun accord de ce type ne fut toutefois signé avec la Pologne. Parfois même des accords bilatéraux postérieurs à la Première Guerre Mondiale empêchaient toute expulsion. Les accords signés en 1920 et 1921 par l'Allemagne avec la République soviétique auraient ainsi interdit toute d'expulsion hors d'Allemagne de personnes s'y trouvant avant la paix de Brest-Litovsk ${ }^{10}$. Le gouvernement allemand poursuit, depuis le début des années 1990, une politique cherchant à établir des accords de réadmission avec le plus grand nombre de pays possibles. L'idée centrale semble être qu'il y aurait des « lacunes » migratoires à combler par le biais d'accords de réadmission avant tout avec les pays d'origine des migrants mais aussi avec les pays de transit. L'ensemble de ces accords devrait en quelque sorte « faire système » comme en témoigne le préambule de l'accord germanopolonais de mai 1993 sur les flux migratoires : «dans le souci d'établir un système régional et, dans le futur, européen d'accords de réadmission [Rückübernahmeabkommen] ... ». Le Ministère fédéral de l'Intérieur ne dissimule pas ses intentions en la matière puisqu'il annonçait à la fin 1994 que «le gouvernement fédéral [avait] l'intention de conclure des accords de réadmission bilatéraux non seulement avec ses voisins immédiats mais aussi avec tous les principaux pays d'origine de demandeurs d'asile " ${ }^{11}$. L'objectif n'est pas seulement de pouvoir procéder à l'expulsion des ressortissants d'un pays mais aussi de dissuader les «entrepreneurs " de flux migratoires illégaux que sont les passeurs ${ }^{12}$, comme l'indiqua le Ministre fédéral de l'Intérieur lors de l'accord avec le Vietnam, soulignant le caractère préventif et dissuasif qu'il attribue à ce type d'accord. Les résultats obtenus avec certains de ces récents accords de réadmission semblent montrer les limites d'une telle stratégie. L'accord conclu en juillet 1995 avec le Vietnam a ainsi permis l'expulsion hors d'Allemagne de 35 ressortissants vietnamiens en 1995 alors que l'accord prévoyait, pour cette même période, l'expulsion de 2500 personnes $^{13}$. De la même façon l'accord d'expulsion des Kurdes conclu avec la Turquie en mars $1995^{14}$ ne semble pas avoir été, dans un premier temps, beaucoup utilisé par les Länder allemands. Au 20 septembre 1995 un seul Kurde avait été expulsé dans ce cadre par les Länder, 27 demandes en ce sens avaient été faites et 14 avaient reçu une réponse de la Turquie indiquant que rien n'était retenu contre eux d'après le droit pénal turc. Ce type d'accord, relativement informel, pourrait toutefois se développer à l'égard de pays que l'Allemagne reconnaît 
avec quelque réserve comme des pays d'origine sûrs, comme c'est le cas pour ceux indiqués aux termes de l'article 29a de la loi sur les procédures d'asile. Les négociations bilatérales en matière d'accords de réadmission demeurent aujourd'hui difficiles. Elles contribuent de plus à brouiller davantage l'articulation interne/externe car le maître d'œuvre de la négociation est le Ministère fédéral allemand de l'Intérieur. L'Auswärtiges Amt ne semble parfois représenter qu'une force d'appoint à la volonté du ministre de l'Intérieur ${ }^{15}$. Définie par David Baldwin comme "toutes les mesures économiques par lesquelles les décideurs de politique étrangère peuvent essayer d'influencer d'autres acteurs internationaux $"{ }^{16}, l^{\prime}$ '« economic statecraft » est au cœur de la «stratégie de pouvoir » choisie par le gouvernement fédéral allemand pour faire fonctionner le dispositif d'expulsion. Les leviers économiques sont en effet les instruments, les techniques les plus souvent utilisées, parmi «l'ensemble des moyens mis en œuvre pour faire fonctionner ou pour maintenir [ce] dispositif de pouvoir ${ }^{17}$. Un véritable bargaining (marchandage) se met en place où l'objectif de rejet d'un groupe d'étrangers (Allemagne) est compensé financièrement directement (budget allemand) ou indirectement. Du coup des acteurs supplémentaires sont impliqués dans le jeu diplomatique déjà complexe ${ }^{18}$, à l'instar, par exemple, des entreprises allemandes voulant investir au Vietnam. Le gouvernement fédéral allemand s'emploie toutefois à démentir tout lien entre ses aides financières à des pays d'origine ou de transit et les accords de (ré)admission conclus avec ces mêmes Etats ${ }^{19}$. On assiste à un accaparement croissant de la diplomatie allemande avec la question de la réadmission, de l'expulsion d'étrangers déclarés indésirables en Allemagne. Le refus des gouvernements d'Erythrée et d'Ethiopie de réadmettre leurs ressortissants renvoyés contre leur gré était ainsi au centre des visites effectuées, début 1996, par le Président fédéral allemand, Roman Herzog, et le Ministre fédéral des Affaires étrangères, Klaus Kinkel, dans la Corne de $l^{\prime}$ Afrique $^{20}$. Il est probable que, pour lever ce refus, le gouvernement puisse utiliser l'aide au développement qu'il verse à ces deux pays (pour l'Erythrée, elle s'élève à 44 millions de DM pour 1995/96). Inversement, l'Erythrée peut, par exemple, dans un semblable marchandage choisir de ne pas conclure un traité bilatéral de protection des investissements ou de trafic aérien (la Lufthansa étant la seule compagnie européenne proposant des vols directs à destination de l'Erythrée). L'accord de réadmission conclu avec le Vietnam contient également plusieurs des éléments de ce type de relation de pouvoir: en 1994, le gouvernement fédéral allemand avait suspendu son aide au Vietnam après que le gouvernement de ce dernier ait refusé de reprendre ses ressortissants expulsés d'Allemagne. En contrepartie de l'acceptation par le Vietnam de reprendre ses ressortissants expulsés, l'Allemagne versera chaque année, en 1995 et en 1996, 100 millions de DM d'aide au développement. Il apparaît cependant que cet " economic statecraft", à l'instar du pouvoir défini par Foucault, est bien plus un " rapport de forces " ${ }^{21}$ qu'une simple allocation de pouvoir à l'un des deux acteurs étatiques. Si le gouvernement fédéral allemand entend inciter par des avantages financiers d'autres Etats-nations à réadmettre leurs ressortissants ou des citoyens d'un pays tiers, il faut considérer que ces Etats disposent également de leviers économiques (l'attribution d'un contrat, la garantie d'investissements allemands...) dans leur relation de pouvoir à l'Allemagne. De plus, d'autres forces, d'autres acteurs sont insérés dans le dispositif d'expulsion, autant d'actions sur des actions dont le contrôle est toujours plus improbable.

Multiplication d'acteurs Le dispositif d'expulsion est une configuration qui rassemble des acteurs hétérogènes, aux capacités d'action et aux statuts fort différents. Le 
caractère transnational du dispositif d'expulsion implique toutefois que tous ces acteurs sont "projetés " sur la scène politique internationale, sans qu'ils aient euxmêmes défini leur rôle à ce niveau. Les organisations non-gouvernementales (ONG) actives au sein du dispositif sont de deux types. A un niveau local, il existe une myriade d'associations humanitaires, à base ethnique ou non, qui s'emploient à empêcher l'expulsion et/ou le placement en centre de rétention d'étrangers (Cf. par exemple les divers « Conseils des réfugiés » [Flüchtlingsräte] fédérant les ONG locales dans plusieurs Länder allemands). Cela prend parfois la forme de contacts directs avec le pays d'origine afin de rassembler des documents attestant d'une persécution, des risques encourus en cas d'expulsion vers ce pays...etc. Elles utilisent le conseil et les informations collectées par des ONG de dimension transnationale (comme Amnesty International). Ces dernières comptent elles-mêmes sur les ONG humanitaires locales pour les informer de pratiques et décisions nouvelles des agences gouvernementales en matière d'expulsion. Cependant les ONG ne sont pas forcément considérées par le gouvernement allemand ou les autorités locales des étrangers comme des adversaires. Leur recours peut permettre de déléguer des tâches difficiles ou coûteuses à accomplir pour les agents étatiques. Mais surtout cela peut laisser espérer une légitimation extérieure du dispositif d'expulsion. Le gouvernement allemand a ainsi cherché à obtenir la caution d'une ONG turque des droits de l'homme (IHD : Association Turque des Droits de l'Homme) qu'il entendait charger de vérifier le respect, par les autorités turques, de l'accord sur le respect des droits de l'homme dans le cas de l'expulsion de ressortissants turcs condamnés en Allemagne pour délits en liaison avec le PKK ou d'autres organisations terroristes (10 mars 1995). IHD, qui aurait été chargée du contrôle du bon traitement des expulsés, a toutefois fini par refuser cette proposition du gouvernement allemand. Dans ce contexte, il est important de souligner le rôle des Eglises allemandes. Fonctionnant comme refuges de demandeurs d'asile déboutés démunis de tout recours juridique supplémentaire, plusieurs paroisses chrétiennes ont défié des arrêtés d'expulsion et soustrait les "expulsables " à une arrestation et un renvoi. Ce type d'action dénommé Kirchenasyl a achevé d'installer ces organisations religieuses au cœur du dispositif d'expulsion, imitant des actions similaires effectuées aux Etats-Unis à la fin des années 1980 en faveur de réfugiés centro-américains. Au niveau supranational mais néanmoins intra-étatique, il est intéressant de remarquer qu'un semblable souci de "délégation" de son autorité sur les exécutions des expulsions a poussé, à l'automne 1995, le Ministère fédéral allemand de l'Intérieur à évoquer l'octroi à l'Organisation Internationale des Migrations (O.M.I.) de la coordination des expulsions, hors d'Allemagne, des demandeurs d'asile déboutés ${ }^{22}$. Par ailleurs les institutions juridiques jouent un rôle prépondérant dans le dispositif. Non seulement leurs décisions a des implications transnationales (par exemple, le refus ou l'autorisation d'expulser vers un pays précis, sur la base de son respect des droits de l'Homme) mais pour rendre ces mêmes jugements il leur faut le plus souvent se plonger dans des recherches complexes pour lesquelles elles ne disposent pas des ressources nécessaires (à l'inverse du Ministère allemand des Affaires étrangères). Le rôle joué par le Tribunal constitutionnel fédéral allemand de Karlsruhe est ainsi à souligner : le sursis d'expulsion, prononcé par ce tribunal le 9 février 1995 envers un ressortissant turc, en raison du danger non complètement exclu qu'il encourrait en rentrant en Turquie, a probablement accéléré la conclusion de l'accord entre les Ministères allemand et turc de l'Intérieur puisque qu'à peine deux semaines plus tard (23 mars) le Tribunal suspendait toute nouvelle décision envers ce plaignant afin de vérifier si le nouvel 
accord pouvait lui être appliqué. Un semblable rôle a été joué par le tribunal dans le cas de sept soudanais assujettis à un arrêté d'expulsion. En 1995, 9 soudanais, qui avaient voyagé de Khartoum vers Moscou et de là vers Francfort sur le Main où ils avaient déposé leur demande d'asile en juillet, étaient maintenus dans la zone de transit suivant les règles des demandes d'asile en aéroport en vigueur depuis juillet 1993. 7 avaient vus leur demande déboutée comme "manifestement infondée ». Pour éviter leur expulsion ordonnée ils entamèrent en août une grève de la faim. Suite à un recours devant le Tribunal constitutionnel fédéral, ce dernier leur accorda un sursis à leur expulsion, alors qu'ils se trouvaient déjà dans l'avion, afin d'examiner la requête et charger le Tribunal administratif de Francfort de vérifier si la grève de la faim pouvait les soumettre à des mesures répressives à leur retour au Soudan. Cette décision fut ouvertement critiquée par le Ministre fédéral allemand de l'Intérieur, qui y voyait une remise en cause d'un des éléments centraux de la réforme du droit d'asile de 1993 : le règlement des aéroports [Flughafenregelung] ${ }^{23}$. Pourtant le Tribunal constitutionnel fédéral leva peu de temps après son interdiction d'expulsion, sur la base d'un écrit du Ministère soudanais des affaires étrangères assurant que les expulsés seraient bien traités dans leur pays d'origine. La cour de Karlsruhe devint ainsi également juge d'un régime étranger et de sa crédibilité en matière de droits de l'Homme, alors que la plupart des ONG soulignaient justement les violations répétées de ces mêmes droits par le régime soudanais. L'expulsion fut de nouveau repoussée début septembre: la Lufthansa ayant fait sortir les Soudanais de l'avion pour la sécurité du vol. D'après la compagnie aérienne, ils auraient commencé à endommager l'avion. En mai 1996, le Tribunal constitutionnel fédéral a reconnu la constitutionnalité de l'essentiel de la réforme sur le droit d'asile de 1993, notamment les clauses concernant les Etats d'origine sûrs et celles liées aux pays tiers sûrs. Les accords bilatéraux de réadmission n'impliquent pas non plus seulement quelques officiels rompus et formés à ces exercices diplomatiques. Lorsque la possibilité d'expulser devient pour un Etat un impératif prioritaire au détriment d'autres intérêts, il n'est pas rare alors que les représentants de ces intérêts choisissent d'atténuer la rigueur de ces priorités. La Chambre allemande du Commerce et de l'Industrie se félicita ainsi de l'accord de réadmission signé avec le Vietnam, y voyant une ouverture pour " une normalisation nécessaire urgente des relations économiques avec ce pays asiatique important » ${ }^{24}$. La conclusion rapide d'un accord de réadmission avec le régime de Hanoï ne devait en effet pas seulement permettre au gouvernement allemand de procéder à l'expulsion de ressortissants vietnamiens mais aussi aux investisseurs allemands de participer aux appels d'offre occidentaux dans la région, qualifiée de «marché doté d'un potentiel de développement énorme ${ }^{25}$. La Bundesgrenzschutz (B.G.S.), la police fédérale allemande des frontières, est l'acteur principal de l'exécution des expulsions. Bien que la compétence en matière d'expulsion (en ce qui concerne les étrangers, en général) est essentiellement laissée aux Länder, la B.G.S. est en fait impliquée dans la plupart des démarches liées à la décision d'expulsion, et ce, au titre de l'aide administrative aux régions. La B.G.S., dont le siègle est situé à Coblence, est ainsi l'institution chargée du contact avec les ambassades étrangères en Allemagne (voire les gouvernements étrangers directement) afin de fournir aux personnes placées en centre de rétention des documents d'identité permettant leur expulsion. La B.G.S. « gère » donc une grande part des informations collectées sur et auprès de la personne à expulser. Elle a fait à plusieurs reprises l'objet de critiques de la part d'organisations humanitaires pour la transmission aux pays de réadmission de données sur les personnes expulsées. Si 
l'expulsion est effectivement exécutée, ce sont des agents de la B.G.S. qui "accompagnent» la personne expulsée jusqu'à son pays de renvoi. Cet « accompagnement » est d'un coût croissant et renseigne, indirectement (à défaut de statistiques officielles régulières), sur le nombre d'expulsions par avion auxquelles les différentes autorités allemandes procèdent. «frais pour voyages de service à l'étranger » : accompagnement des expulsés par la Bundesgrenzschutz

Coûts effectifs (millions de DM) ${ }^{26}$ Coûts prévus au budget fédéral (millions de DM) 1992 5,272 1993 8,409 4,495 1994 12,114 71995 8,5 1996 12,5 Enfin, la Bundesgrenzschutz, agence chargée du contrôle des frontières, est responsable de l'ensemble des expulsions immédiates effectuées dans les zones frontalières vers les pays voisins. La B.G.S. est en définitive le véhicule principal de la transnationalisation croissante du Ministère fédéral allemand de l'Intérieur, négociant souvent avec les autorités de pays étrangers sans l'intermédiaire du Ministère allemand des Affaires étrangères. La dernière étape $\mathrm{du}$ processus d'expulsion implique enfin des acteurs privés disposant d'une marge de décision non négligeable sur l'exécution de l'expulsion : les compagnies aériennes. Elles sont d'ailleurs, en amont, tenues pour responsables de toute entrée illégale d'étrangers ayant utilisé leurs services. Si elles ont omis d'effectuer les contrôles nécessaires, elles sont obligées de supporter les coûts liés, le cas échéant, à l'expulsion de la personne. Le commandant de bord d'un avion, première instance à bord, peut choisir de refuser de transporter un expulsé, s'il considère que l'expulsion met en péril la sécurité des autres passagers. Il semble que les exigences vis-à-vis de la Bundesgrenzschutz varient suivant les compagnies aériennes. Les expulsions seraient même devenues "un facteur économique" pour les compagnies aériennes, ces dernières devant généralement émettre trois billets pour chaque individu expulsé (deux agents de la B.G.S. l'accompagnant en général). Des tarifs préférentiels seraient même consentis au personnel "d'accompagnement " ${ }^{27}$. Les relations de pouvoir qui sont à l'œuvre au sein du dispositif d'expulsion en Allemagne ne sont pas (ou plus) limitées à un champ fermé, se caractérisant par des lieux, des acteurs spécifiques. Elles constituent un espace propre d'influence, de pression, de négociation avec deux extrêmes : d'une part la relation de pouvoir entre chaque individu sujet à l'expulsion et les agences de contrôle qui tentent de réaliser cette expulsion. D'autre part, la relation de pouvoir (inter-/trans-étatique) entre les Etats souhaitant procéder à l'expulsion et ceux devant l'accepter. Ces deux pôles mobilisant des ressources fort différentes partagent toutefois un semblable impératif de résistance: l'ultime résistance d'un individu dans le dénuement et le refus de parler, de se laisser voir et la résistance (parfois atténuée en "réticence ») d'Etats à réadmettre leurs ressortissants ayant migré illégalement vers un autre Etat.

Revenir à la partie précédente

Revenir à la partie précédente 


\section{NOTES}

1. En 1816, 1830, 1844, 1857 et 1872 . Ces "Kartellkonvention" revêtaient déjà à l'époque une grande importance. Le gouvernement prussien procéda ainsi, entre septembre 1842 (date de l'expiration de la convention de 1830) et mai 1844 (signature de la nouvelle convention) à des mesures unilatérales afin de contrôler l'immigration de Juifs et de Polonais, à laquelle il ne pouvait plus opposer des expulsions (leur fondement légal -la convention prusso-russe - n'étant plus en vigueur).

2. Cf. Neubach, op. cit., p. 6 et 37.

3. Sur ce thème voir surtout l'ouvrage de Didier Bigo, Polices en réseaux : l'expérience européenne, Paris, Presses de Sciences-Po, 1996.

4. La Pologne a, par exemple, conclu des accords de réadmission avec l'Ukraine, la Roumanie, la Slovaquie et la Bulgarie.

5. Au premier semestre 1993 les ressortissants roumains représentaient $41 \%$ de l'ensemble des étrangers entrés illégalement en Allemagne, au second semestre, après la réforme constitutionelle sur le droit d'asile et l'adoption de la notion de pays d'origine "sûrs", ils en représentaient $23 \%$.

6. Entre l'entrée en vigueur de l'accord de réadmission germano-bulgare (entre le 9 septembre 1994 et avril 1995) 1919 ressortissants bulgares ou présumés tels ont été expulsés par avion d'Allemagne (soit en moyenne près de 240 personnes par mois) , 734 supplémentaires ont été "renvoyés" [zurückgeschoben] - selon $\$ 61$ de la loi sur les étrangers - dans les 6 mois suivants leur entrée illégale en Allemagne (soit en moyenne 92 personnes par mois). Cf. Deutscher Bundestag, 13. Wahlperiode, Drucksache $13 / 1605 ;$ p.6.

7. Depuis l'entrée en vigueur de l'accord de réadmission germano-roumain (entre le 1er novembre 1992 et avril 1995) 38919 ressortissants roumains ou présumés tels ont été expulsés par avion d'Allemagne (soit en moyenne plus de 1300 personnes par mois), 32001 supplémentaires ont été "renvoyés" dans les 6 mois suivants leur entrée illégale en Allemagne (soit en moyenne plus de 1100 personnes par mois). Source : voir note précédente.

8. Cf. Maurer, op.cit., pp.355-357.

9. Cf. Maurer, op.cit., p.392.

10. Cf. Maurer, op.cit., p.425.

11. Innenpolitik, nr. VI, 19.12.1994.

12. Cf. "Abkommen über Rückübernahme von Vietnamesen", ZAR Aktuell, $n^{\circ} 5,25$ septembre 1995.

13. Cf. "Deutschland und Vietnam vereinbaren Zusammenarbeit", Frankfurter Allgemeine Zeitung, 29 février 1996.

14. L'accord ne serait entré en vigueur qu'au 17 juillet 1995.

15. Cf. dossier des Soudanais.

16. David Baldwin, Economic Statecraft, Princeton, 1985, p.40.

17. Définition par Michel Foucault de la "stratégie de pouvoir" : Michel Foucault, "Le pouvoir, comment s'exerce-t-il ?", op.cit., p.319).

18. Il faut également tenir compte des rivalités et des objectifs divergents des différents ministères fédéraux : les Ministères fédéraux des Affaires étrangères et de l'Economie se sont ainsi opposés au souhait du Ministère fédéral de l'Intérieur d' établir un lien 
direct entre l'accord de réadmission avec le Vietnam et le programme allemand de garantie des investissements (équivalents des crédits COFACE en France). Cf. "Kohl verlangt von Hanoi die Erfüllung bestehender Vertragsverpflichtungen", Frankfurter Allgemeine Zeitung, 20 novembre 1995, p.2.

19. Voir les propos du Secrétaire d'Etat Kurt Schelter in Deutscher Bundestag, 13. Wahlperiode, Drucksache 13/1605; p.6.

20. Cf. "Gespräche über Rückführung abgelehnter Asylbewerber geplant", Frankfurter Allgemeine Zeitung, 31 janvier 1996, p.5.

21. Cf. Gilles Deleuze, Foucault, Paris, Editions de Minuit, 1986, notamment pp.77-99 : "Les stratégies ou le non-stratifié : la pensée du dehors (pouvoir)" : « Qu'est-ce que le Pouvoir? La définition de Foucault semble très simple, le pouvoir est un rapport de forces, ou plutôt tout rapport de forces est un « rapport de pouvoir ». Comprenons d'abord que le pouvoir n'est pas une forme, par exemple la forme-Etat; et que le rapport de pouvoir n'est pas entre deux formes, comme le savoir. En second lieu, la force n'est jamais au singulier, il lui appartient essentiellement d'être en rapport avec d'autres forces, si bien que toute force est déjà rapport, c'est-à-dire pouvoir : la force n'a pas d'autre objet ni sujet que la force (...) Foucault est plus proche de Nietzsche (et de Marx aussi), pour qui le rapport de forces excède singulièrement la violence, et ne peut se définir par elle. C'est que la violence porte sur des corps, des objets ou des êtres déterminés dont elle détruit ou change la forme, tandis que la force n'a pas d'autre objet que d'autres forces, pas d'autre être que le rapport : c'est « une action sur l'action, sur des actions éventuelles, ou actuelles, futures ou présentes ", c'est un ensemble d'actions sur des actions possibles ». On peut donc concevoir une liste, nécessairement ouverte, de variables exprimant un rapport de forces ou de pouvoir, constituant des actions sur des actions : inciter, induire, détourner, rendre facile ou difficile, élargir ou limiter, rendre plus ou moins probable.... Telles sont les catégories de pouvoir. » 22. "Will Innenministerium Abschiebungen delegieren? / Unklare Absichten", Frankfurter Allgemeine Zeitung, 11.12.1995.

23. "Kanther kritisiert das Bundesverfassungsgericht", Frankfurter Allgemeine Zeitung, 26.08.1995, p.1.

24. Cf. "Kabinett billigt Rückführungs-Abkommen mit Vietnam", Süddeutsche Zeitung, 12.01.1995.

25. Propos de Bernd Gottschalk (Mercedes-Benz AG), "Montage in Vietnam beginnt im nächsten Jahr", Frankfurter Allgemeine Zeitung, 21.11.1995. Le choix de Mercedes d'investir 70 millions de dollars au Vietnam, surtout dans la production de véhicules utilitaires, est intervenu quelques semaines après l'accord de réadmission.

26. [Il faut noter que les dépenses supplémentaires sont censées être financées par les recettes de la BGS issues du remboursement par l'O.N.U. de la participation de la BGS à des missions de paix de l'O.N.U.].

27. Cf. Knösel, op.cit., pp.76-77. 
INDEX

Mots-clés : étrangers, expulsions, contrôle

Index géographique : Allemagne 